\begin{tabular}{|l|l|l|l|}
\hline Eiszeitalter $u$. Gegenwart & 38 & $\begin{array}{l}17-36 \\
7 \mathrm{Abb} .\end{array}$ & Hannover 1988 \\
\hline
\end{tabular}

\title{
Quartäre Vereisungen in den lothringischen Vogesen: Anzahl, Ausdehnung und Alter
}

\author{
Jean-Claude Flageollet *)
}

Ice-marginal features, moraine, glaciofluvial sedimentation, glaciolacustrine sedimentation, periglacial environment, fluviolacustrine sedimentation, stratigraphy, lithofacies

France, Vosges Mountains

Kurzfassung: Die abschließende Auswertung der Arbeiten zur Aufnahme der Geologischen Karte 1: 50.000 der Vogesen (Blätter BRUYERES und EPINAL) hat bestätigt, daß der mittlere Teil der westlichen Vogesen von zumindest drei aufeinanderfolgenden Vereisungen betroffen war.

Vor allem die Randlagen der dritten Vereisung sind in den Tälern von Mosel, Ognon und Moselotte und im Gebiet um Le Tholy nachgewiesen.

Unter Bezug auf die für Grand Pile und Les Echets aufgestellten Stratigraphien kann man die dritte Vereisung des Moselbeckens dem Würm-Hochglazial zuordnen. Daraus ergibt sich mindel- bzw. rißzeitliches Alter für die vorausgehenden Vereisungen.

\section{[Quaternary Glaciations in the Vosges in Lorraine:} Times of Occurence and Extent]

Abstract: At the conclusions of the investigations made in view to work out the last geological maps at $1 / 50000$ scale in the Vosges (sheets BRUYERES, EPINAL) one can confirm that the middle western Vosges were affected by at least three successive glaciations.

The boundaries of each, mainly those of the third one, are classified in the Moselle, Ognon and Moselotte valleys, as well as in the Le Tholy area.

In reference to the chronologies drawn up at La Grande Pile and les Echets, we are allowed to give an age Würm-Pleniglacial to the third glaciation in the Moselle basin and, therefore, an age Mindel and an age Riss to the previous ones.

[Glaciations quaternaires dans les Vosges lorraines: Nombre, extensions et âges]

Résumé: A la fin des recherches que nous avons effectuées pour dresser les dernières cartes géologiques à I/ 50000 des Vosges (feuilles BRUYERES, EPINAL) nous pouvons confirmer

*) Anschrift des Autors: Prof. Dr. J.-C. Flageollet, Centre de Recherches en Géographie Physique de l'Environment, Université de CAEN - 14032 CAEN Cedex. France. que les Vosges moyennes lorraines ont connu au moins trois glaciations successives.

Les limites de chacune de ces glaciations, principalement celles de la troisième, sont précisées dans les vallées de la Moselle, de l'Ognon et de la Moselotte, ainsi que dans le secteur du Tholy.

En se référant aux chronologies établies à la Grande Pile et aux Echets, nous sommes fondés à donner un âge pléniglaciaire du Würm à la troisième glaciation dans le bassin de la Moselle et, par conséquent, à placer les deux précédentes au Mindel et au Riss.

\section{Einführung}

Im Vergleich mit der bescheidenen Größe der Vogesen gibt es eine beachtliche Zahl von Autoren und Arbeiten, die sich mit der Frage quartärer Vereisungen in diesem Raum beschäftigen (FLAGEOLLET: 1984). Man hat zwar bereits einen gewissen Kenntnisstand erreicht, es bleiben aber noch eine ganze Reihe von Diskussionspunkten sowie Unterschieden oder gegenläufigen Meinungen in der Interpretation bestehen. Dies gilt insbesondere für die Bereiche, die am stärksten von Forschern unterschiedlicher Nationalität und unterschiedlicher Schulen untersucht worden sind, wobei das eine möglicherweise das andere erklärt. Für die Vogesen nördlich des Col de Bonhomme wie auch für die elsässische Abdachung der mittleren und südlichen Vogesen fehlen derartige Kontroversen oder sind zumindest weniger heftig. Im Gegensatz dazu waren sie in den letzten zwanzig Jahren für den Bereich der mittleren und südlichen Vogesen westlich einer Linie Ballon d'Alsace/Col de Bonhomme sehr lebhaft. Syntheseversuche, die für die Ausarbeitung der Geologischen Karte von Frankreich 1:250.000 unternommen wurden, stoßen sich an unterschiedlichen Interpretationen hinsichtlich der Zahl der Vereisungen, ihrer jeweiligen Ausdehnung und ihrer Stellung innerhalb der Quartärstratigraphie. 
Damit beauftragt, in Zusammenarbeit mit P. L. VINCENT quartäre Bildungen in verschiedenen Teilen des Moselbeckens für die Geologische Karte von Frankreich 1:50.000 aufzunehmen - für Lothringen die Blätter Nancy, Toul und Briey, für die Vogesen die Blätter Munster und Gérardmer p. p., Remiremont, Bruyeres und Epinal - oblag mir eine zweifache Aufgabe, wie sie für Kartierungen dieses Maßstabs üblich ist:

Die Aufnahme aller Ablagerungen nach Lage und Umfang einerseits und die Herstellung von Zusammenhängen mit Befunden benachbarter Kartenblätter andererseits. Bei diesem häufig heiklen, jedoch stets anregenden Unterfangen wird die Forschern vertraute Versuchung gebremst, einen Nachweis auf gerade ihrer Deutlichkeit wegen ausgewählte Bildungen zu stützen und dabei die problematischen beiseite zu lassen, was aber hieße, einige in begrenzten Bereichen gewonnene Schlußfolgerungen zu generalisieren.

Ausgehend von Beobachtungen und kartographischen Aufnahmen zwischen Remiremont und Epinal wird im folgenden zunächst die Frage nach der Zahl der Vereisungen diskutiert. Die Frage nach der Ausdehnung der letzten Vereisung und ihrer Spätphasen wird anhand dreier Stellen erörtert: Dem Tal der Cleurie und den oberen Talabschnitten von Ognon, Moselotte und Vologne. Der dritte Abschnitt behandelt das Problem des Alters der Vereisungen durch Vergleich der morphologischen Befunde mit den Pollenanalysen aus dem Torfmoor von Grand Pile.

\section{Existenz und Randlagen mindestens dreier aufeinanderfolgender Vereisungen zwischen Remiremont und Epinal (Abb. 1)}

Erratische Kristallinblöcke, im wesentlichen Granite, die auf den Sandsteinplateaus liegen, und ein unzusammenhängendes Pflaster von Moränenresten sind die Hauptzeugen der Ausdehnung des oder der ersten Eisvorstöße in diesem Teil der Vogesen. Dazu treten Sedimente, die heute als zerschnittene Terrassen unterhalb von Epinal gefunden werden.

\subsection{Es gab wenigstens eine ältere Vereisung (W)}

1.1. Im Westen ist ihre Ausdehnung vom Raum Plombières-les-Bains bis Epinal und vom Gebiet um Epinal bis Corcieux bestimmbar. Nach den Vereisungsspuren hatten die Gletscher niemals den Hang überfahren (Steilstufe zusammengesetzt aus Bruchwänden und Bruchlinienstufen), der südlich des Horstes von Epinal endet und sich entlang einer Linie Uriménil, Laménil und Charmois devant Bruyeres erstreckt. Abgesehen von den fluviatilen Sedimenten und den Terrassen X und Y (s. u.) wurden auf dem Horst selbst keinetlei kaltzeitliche Sedimente gefunden. Es scheint deshalb, daß dieser zu keiner Zeit von den quartären Gletschern bedeckt oder erreicht wurde. Jedoch wurden die Granitblöcke, die in den Schotterdecken von Epinal beobachtet wurden, mit der Ausdehnung eines Gletschers bis an die Stelle der heutigen Stadt in Verbindung gebracht (SERET 1967: 418-458). Andererseits hätten bei tektonischer Hebung des Horstes und durch die starke Eintiefung der Nebenbäche des linken und rechten Moselufers in den Horst die auf dem Horst abgelagerten Moränen entfernt werden können. Für diese Hypothese spricht das völlige Fehlen alt-W-zeitlicher Schüttungen zwischen Dinozé und Epinal. M.E. bleibt die Ungewißheit bestehen, aber aus drei Gründen ist die erste Annahme die wahrscheinlichere.

Die alten Sedimente des Bois de la Voivre unmittelbar am Horstrand haben nicht die Merkmale einer Eisrand-Ablagerung. Es wäre überraschend, hätte die Erosion alle Reste glazialer Ablagerungen einschließlich erratischer Blöcke entfernt. Die Granite des Horstes von Epinal sind zu Wollsäcken verwittert

Abb. 1: Quartäre Bildungen im Moseltal zwischen Remiremont und Epinal und ihr Alter.

RG : Vereisungsspuren

GF : Undifferenzierte glaziale Bildungen

$M$ : Moräne

MT: End- oder Stirnmoräne

GL : Glazio-lakustrine Bildung

FG : Fluvio-glaziale Bildung s. s. (innerhalb der letzten Vereisung)

I : Sander

FL : Fluvio-lakustrine Bildung

F Fluviatile Schüttung im eisrandnahen und im periglazialen Bereich

$\begin{array}{ll}\mathrm{W} & : \text { Ältere Vereisung } \\ \mathrm{W} 1, \mathrm{~W} 2: & \text { Erste und zweite ältere Vereisung oder } \\ & \text { Stadien } 1 \text { und } 2 \text { der älteren Vereisung }(\mathrm{W} \chi) \\ \mathrm{W}-\mathrm{X} \quad: \text { Ältere oder „mittlere" Vereisung } \\ \mathrm{X} & : \text { "Mittlere" Vereisung (wahrscheinlich Riß) } \\ \mathrm{X} 1, \mathrm{X} 2: & : \text { Stadien der mittleren Vereisung } \\ \mathrm{X}-\mathrm{Y} & : \text { Mittlere oder jüngere Vereisung } \\ \mathrm{Y} & : \text { Jüngere Vereisung (Würm) } \\ \mathrm{Y} 1 & : \text { Ablagerungen des Hauptvorstoßes } \\ \mathrm{Y} 2, \mathrm{Y} 3 \quad: \text { Erosionsterrassen in den Schüttungen von Y1 } \\ \mathrm{Z} & : \text { Holozän } \\ 1 & : \text { Torf } \\ 2 & : \text { Störung, Verwerfung } \\ 3 & : \text { Höhenlinien im Abstand von } 50 \mathrm{~m}\end{array}$




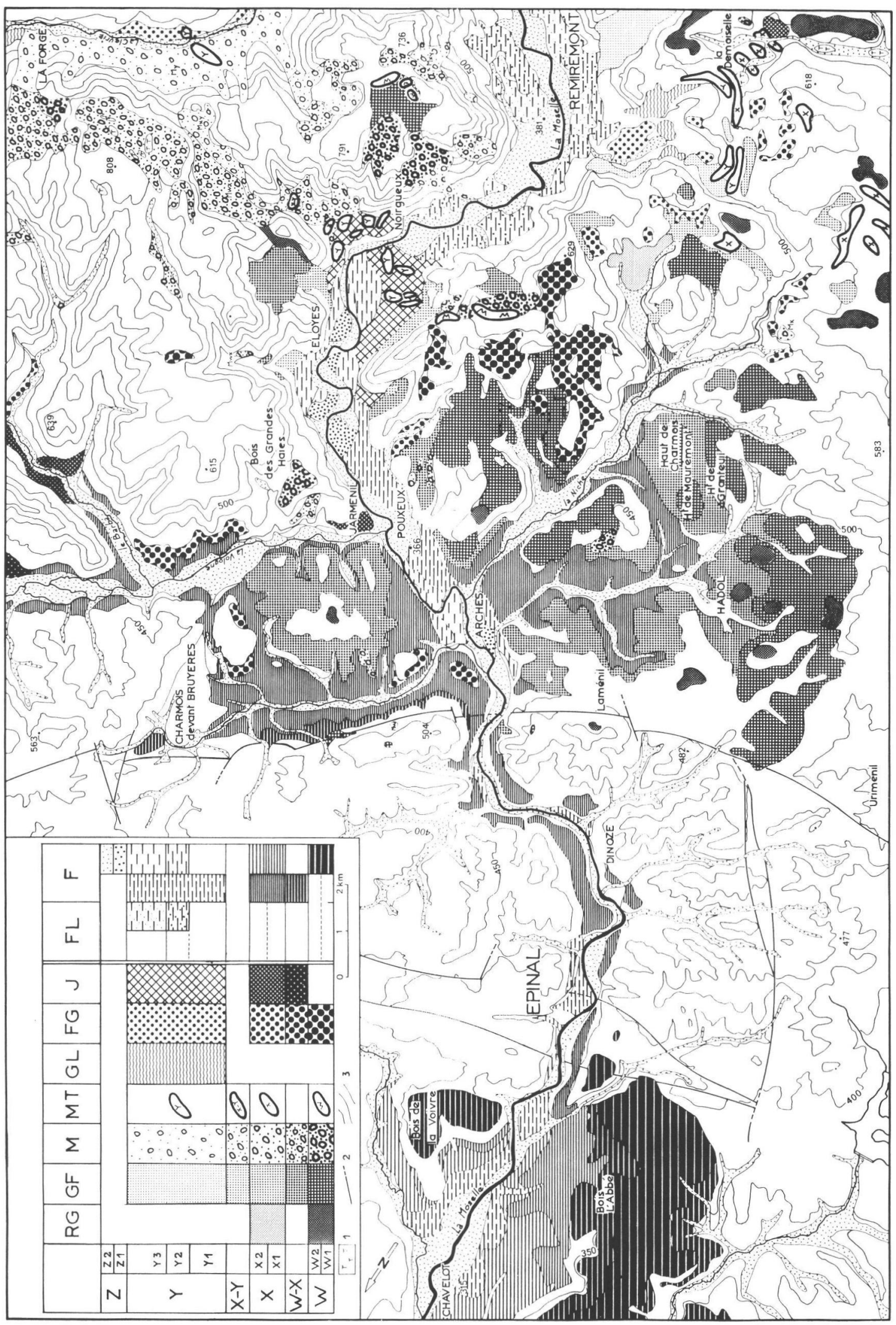


und die, die in den Sedimenten von Epinal eingeschlossen sind, können von den benachbarten Hängen stammen.

Im Moment möchte ich also eher annehmen, daß die älteren Gletscher gegen den Horst gestoßen sind, ohne ihn zu bedecken und ohne tiefer in ihn einzudringen.

\subsection{Gab es zwei Stadien einer einzigen älteren Vereisung oder zwei ältere Vereisungen?}

Diese Frage stellt sich, wenn man die Verbreitung der Ablagerungen zum einen innerhalb der äußersten, oben beschriebenen Eisrandlage, zum anderen unterhalb des Horstes von Epinal überprüft, wo sich zwei ältere Terrassenniveaus befinden.

\subsubsection{Die glazialen Ablagerungen des Beckens von Hadol}

Wenn man dort auch ohne allzu große Schwierigkeiten die quartären Bildungen lokalisieren kann, die die triassischen Sandsteine überdecken, so ist es doch weitaus schwieriger, ihren genauen Ursprung zu bestimmen (Moräne s. s. oder fluvio-glazial) und noch schwerer, eine zuverlässige relative Stratigraphie zu erstellen. Die Mächtigkeit der Ablagerungen und die Morphologie der liegenden Sandsteine sind kaum bekannt. Manche Bereiche zeigen das ebene Bild einer Akkumulationsfläche, aber die Ebenheit ist möglicherweise an das Vorhandensein von Strukturoberflächen oder schichtakkordanten Flächen im liegenden Sandstein gebunden. Bei den Hügeln (Haut de Granteuil, Haut de Maurémont, Haut du Charmois) kann es sich um Stirnmoränen handeln, wie man es im Aufschluß von Raon aux Bois beobachtet, oder um eingeschachtelte, stark aufgelöste, fluvio-glaziale Terrassen unterschiedlichen Alters. Es besteht aber auch die Möglichkeit, daß es sich um einfache Moränen- oder fluvio-glaziale Pflaster handelt, die die Sandstein-Resthügeln umschließen. Aus Mangel an tiefen Aufschlüssen ist die Interpretation ausgehend von der reinen Morphologie heikel. Um sicher entscheiden zu können, sind weitere Untersuchungen nötig. Deshalb haben wir vorerst auf Bezeichnungen wie GF (undifferenzierte Bildungen) und W-X (ältere oder mittlere Vereisung) zurückgegriffen.

\subsubsection{Die hochgelegenen Terrassen unterhalb von Epinal}

Zwischen Epinal und Thaon-les-Vosges wurden ältere Ablagerungen durch die Mosel und die Avière in höhergelegene Terrassen zerschnitten. Diese Sedimente sind von mächtigen Lehmen bedeckt. Wir konnten das Material an zwei Stellen beobachten:

- Im Straßenanschnitt der Ostumgehung von Epinal, im Bois de la Voivre: Die lehmige Überdekkung war hier in ihrer ganzen Mächtigkeit auf 200 m Länge aufgeschlossen.

- Im Anschnitt der Verbindungsstraße zwischen Bois l'Abbé und Chavelot westlich von Epinal.

Ausgehend von ihrer flächenhaften Ausdehnung ist mit einem beträchtlichen Volumen dieser älteren Sedimente zu rechnen. In wesentlich höherem Anteil als den von SERET (1967: 450) angegebenen 0,1\% kommen Granit- und Gneisgerölle der Vogesen häufig in einer sandig-tonigen Matrix vor; weil sie aber stark verwittert und sehr mürbe sind, treten sie an der Oberfläche nicht mehr in Erscheinung. Durch ihr Volumen, die Art und die Größe der Schotter haben diese Sedimente das gleiche Aussehen wie fluvioglaziale Ablagerungen, die der ältesten Vereisung oder den ältesten Vereisungen der Vogesen entsprechen. Diese Frage stellt sich, weil die Untersuchung der Topographie zwei ineinandergeschachtelte Terrassenniveaus ergibt, deren Bedeutung noch unsicher ist:

Handelt es sich um zwei Niveaus in Verbindung mit zwei Vereisungen oder um Terrassen, die nur zwei Stadien einer einzigen älteren Vereisung entsprechen? Die Mächtigkeit der Lehme ist offensichtlich auf beiden Niveaus sehr ähnlich; drei Viertel der Kristallingerölle des unteren sind vollständig verwittert; nur die Paläoböden des oberen Niveaus wurden von CAILLEUX 1977 untersucht, nicht aber die des unteren.

\subsection{Eine zeitlich und räumlich dazwischenliegende Vereisung}

Sie war räumlich wesentlich enger begrenzt als die vorausgehende(n), und die sie belegenden Ablagerungen sind zahlreicher, vollständiger und enthalten insbesondere schöne „End-Komplexe”: Endmoränen, Sanderflächen und Sedimentdecken in den Seitentälern der Mosel, Niche und in noch höherem Maße des Barba. Die Lage dieser Komplexe zeigt ein Überfließen des Eismassen über die Sättel an sowie die Stirn eines Moselgletschers s. s. zwischen Eloyes und Jarménil.

Diese Vereisung wurde von G. SERET erkannt und der Mindel-Eiszeit zugeordnet.

Sie ist es, die die durchgehendste Akkumulationsterrasse hinterlassen hat, in mittlerer Höhenlage zwischen den Talhangoberkanten und den rezenten Talsohlen. Oberhalb des Horstes von Epinal zwischen 


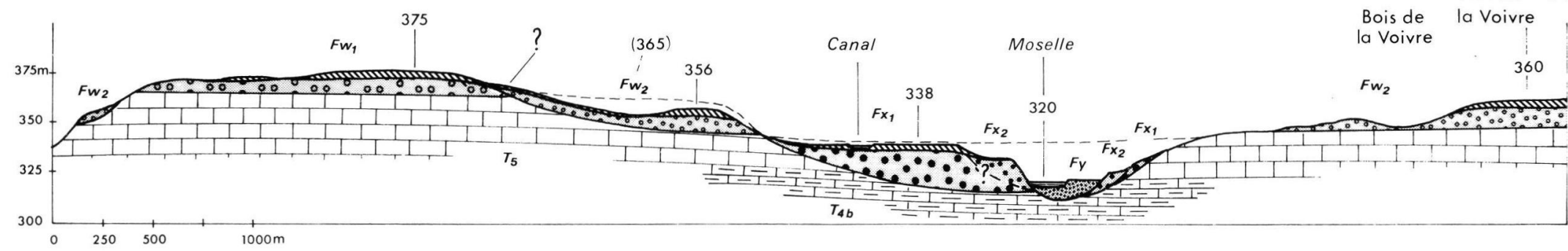

आप Löss

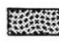

Fluvioglaziale Sedimente, $\boldsymbol{Y}$.Vereisung (WÜRM) $F_{y}$ : Talterrasse

Fluvioglaziale Sedimente, $\boldsymbol{X}$.Vereisung (Riss)

$\because \because \quad$ Fluvioglaziale Sedimente, $X$.

Fx : : Eingeschachtelte Terra

Fluvioglaziale Sedimente, W. Vereisung (Mindel)

$\because \because F_{2}$ : Eingeschachtelte Terrasse

○० $F w_{1}:(55 m-N i v e a u)$, Talterrasse

Abb. 2: Mosel-Terrassen unterhalb von Epinal.

$T_{5}$ : Oberer Muschelkalk 
Hadol und Charmois devant Bruyeres weitet sie sich durch Zusammenfluß von Schmelzwässern des Eisrandes in den Tälern von Barba, Mosel und Niche im Becken von Arches stark aus. Man kann sie innerhalb des Horstes verfolgen, wo sie sehr schmal ist, und jenseits davon, unterhalb von Epinal, wo sie sich von neuem ausweitet; anschließend längs des Tales bis Toul, wo sie die "Mittelterrasse” der Justice bildet (Geologische Karte von Frankreich 1:50.000, Blatt Toul). Die Paläoböden auf dieser Terrasse bei Golbey und bei Archettes wurden von SERET (1967: 439ff.) und von CAILLIER (1977) untersucht.

Die morphologischen Modifikationen, vor allem eine Verengung und bedeutende Eintiefung im Verhältnis zum Vor-W-Tal (Abb. 2), die Körnungs- und mineralogischen Unterschiede der Sedimente und der Lehme und die Paläoböden zeigen klar, daß es sich um eine eigenständige Vereisung handelt.

Diese Unterschiede, vor allem bezüglich der Höhenlage, die in den fluvio-glazialen und den fluviatilen Decken im Arches-Becken (Fw-x und Fx) und unterhalb von Epinal (Fx1-Fx2) festgestellt wurden (Abb. 1), stellen gleichermaßen die Frage nach eventuellen Stadien innerhalb dieser Vereisung.

Man könnte mit SERET an ein Akkumulationstadium während des Hochglazials (oberes Niveau) denken, gefolgt von einer weiteren wichtigen Akkumulation beim Rückzug (SERET 1967: 539ff.). Aber dafür gibt es keine gesicherten Belege. Mehr noch, für die Moräne von Noirgeux haben wir diese Interpretation abgelehnt FlageOliet 1984).

\subsection{Eine dritte Vereisung}

Eine dritte Vereisung ist eindeutig im Moseltal nachgewiesen, wo sich der „End-Komplex” von Noirgeux befindet (Moräne und Sander). Er setzt sich fort in einer Sedimentdecke, die vom Fluß zerschnitten wird. Sie bildet oberhalb und unterhalb des Horstes von Epinal eine niedrige, später sehr niedrige Terrasse. Wir haben in der Tat die Terrasse von Pouxeux als Verlängerung des Sanders von Noirgeux interpretiert (FlageOliet 1984, VASKov 1984). Der Riegel, der durch den gemeinhin „Moräne von Noirgeux” genannten Komplex geschaffen wird, hat recht lange oberhalb einen See aufgestaut, der bis in den Raum Remiremont reichte (SERET 1967: 372; FLAGEOLLET 1984).

\subsection{Zusammenfassung}

Wenigstens drei Gletscher, die aufeinanderfolgenden Vereisungen mit immer geringerer Ausdehnung angehörten, haben das Moseltal ausgefüllt. In diesem Punkt sind wir mit SERET einig. Eine eindeutige
Festlegung der zugehörigen Eisrandlagen in den Nebentälern (Vologne, Moselotte) sowie in den Tälern und auf den Plateaus der Haute-Saone ist noch nicht mit letzter Sicherheit möglich.

\section{Ausdehnung und Rückzugsphasen der letzten Vereisung}

Wir werden hier das Becken von Remiremont (SERET 1967, FLAGEOLLET 1979) und das Volognetal zwischen Granges sur Vologne und Docelles außer acht lassen, wo die Korrelation mit dem Volognetal s. s. große Probleme aufwirft, um uns mit drei Bereichen zu beschäftigen, in denen die Interpretationen am stärksten diskutiert waren und noch sind: dem Cleurietal, der oberen Ognon und der oberen Moselotte.

\subsection{Das Tal der Cleurie}

Die dem Quartär dieses Tales gewidmeten Arbeiten reichen weit zurück und sind zahlreich (MEYER 1913; Nordon 1931; CAPOT-Rey 1938; SERET 1967; SALOME 1968; Flageollet \& Hameurt 1971; Flageollet 1976; 1984).

Über folgende Fragen wollen wir hier schwerpunktmäßig sprechen:

- Zu wievielen verschiedenen Vereisungen oder Vereisungsstadien gehören die Ablagerungen, die hier vorkommen?

- Welcher oder welchen ist der See zuzuordnen, in dem sich das Delta aufgebaut hat, das talabwärts die heute den See von Gérardmer aufstauende Moräne verlängert? Wie groß war seine Ausdehnung und wodurch wurde er aufgestaut?

- Stammen die Ablagerungen von einem Gletscher, der von der Vologne kam und das Tal nach Westen hinabfloß, oder gab es ein Zusammenfließen von Eiszungen, wie SERET es angegeben hat, und wieviele waren es?

\subsubsection{Die zwischen Tholy und dem See von}

Gérardmer nachgewiesenen Ablagerungen stammen von drei Gletschern, die aufeinander zugeflossen sind.

Ohne auf allen Details einzugehen, lassen sich drei Gruppen von Argumenten anführen:

- morphologische Argumente: Es handelt sich um bogenförmige Ablagerungen, deren Auswölbungen zwischen Tholy und Rain Brice nach E, zwischen dem See von Gerardmer und dem Bas Beillard nach W und nach $\mathrm{N}$ in das Tal von Cellet gerichtet sind. 

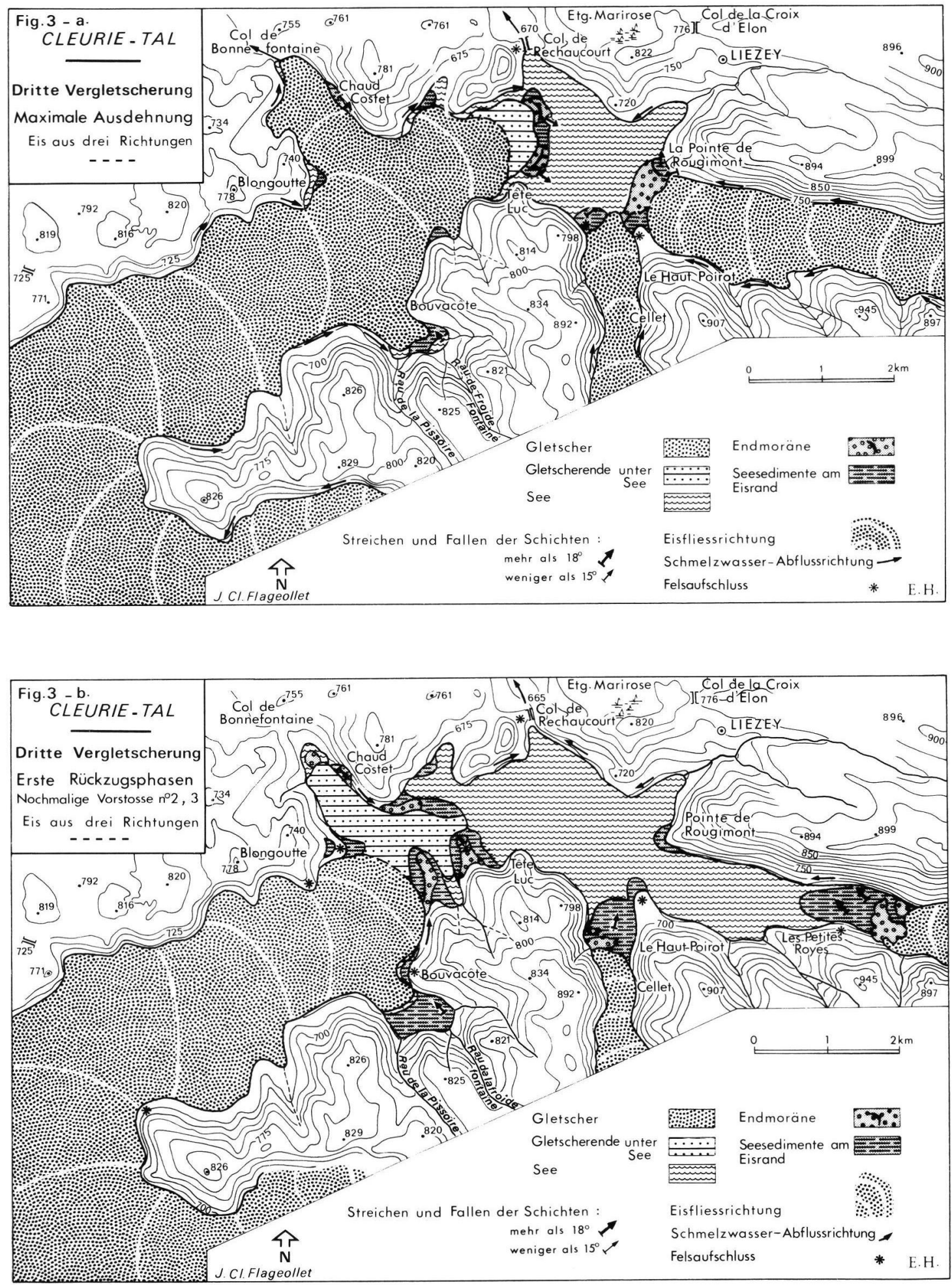

Abb. 3a und b: Cleurie-Tal. Dritte Vergletscherung, maximale Ausdehnung und erste Rückzugsphasen. 

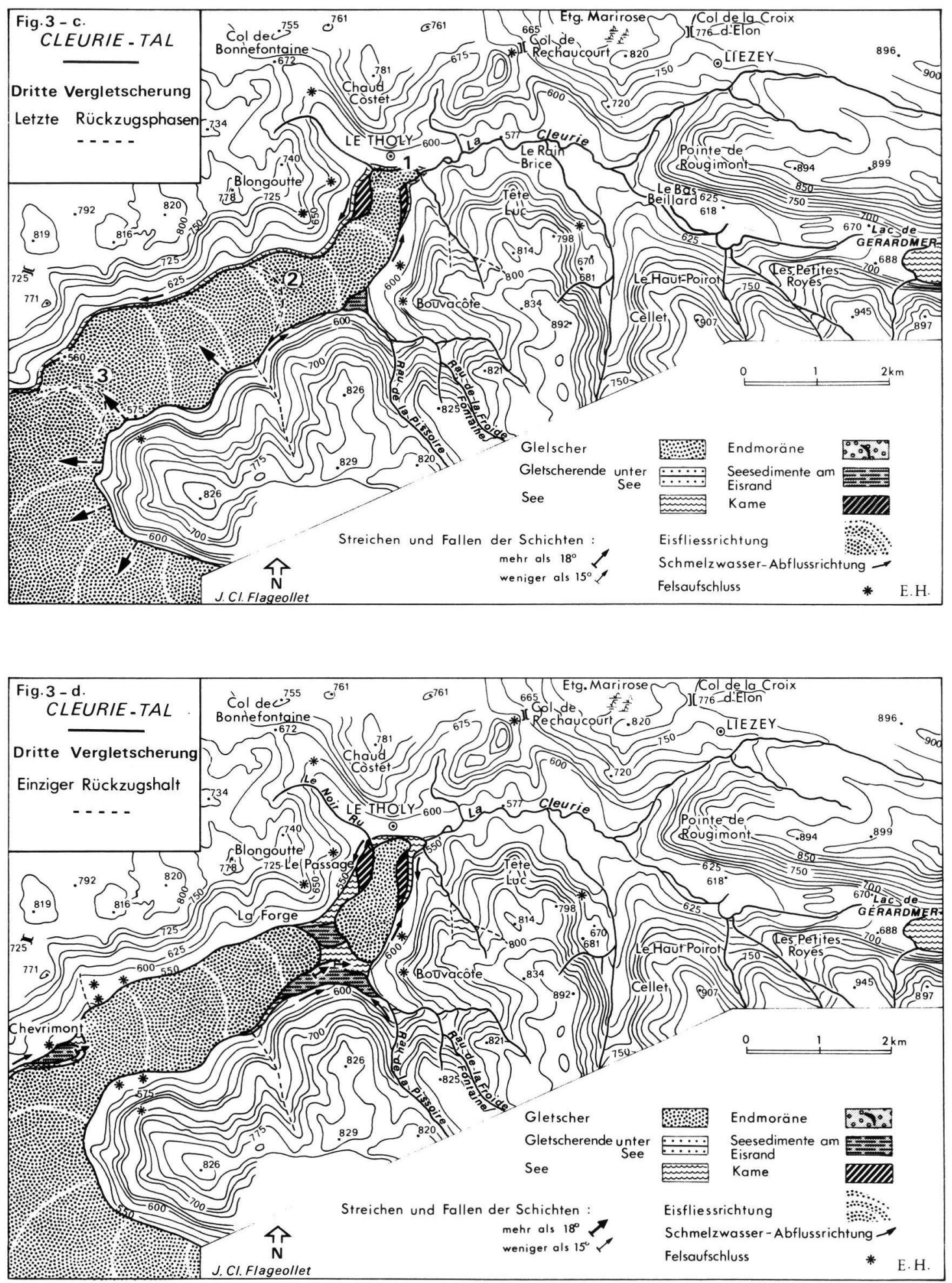

Abb. 3c und d: Cleurie-Tal. Dritte Vergletscherung, letzte Rückzugsphasen und einziger Rückzugshalt.

Abb. 3e: Cleurie-Tal. Dritte und letzte Vergletscherung. Chronologie der Ablagerungen. 


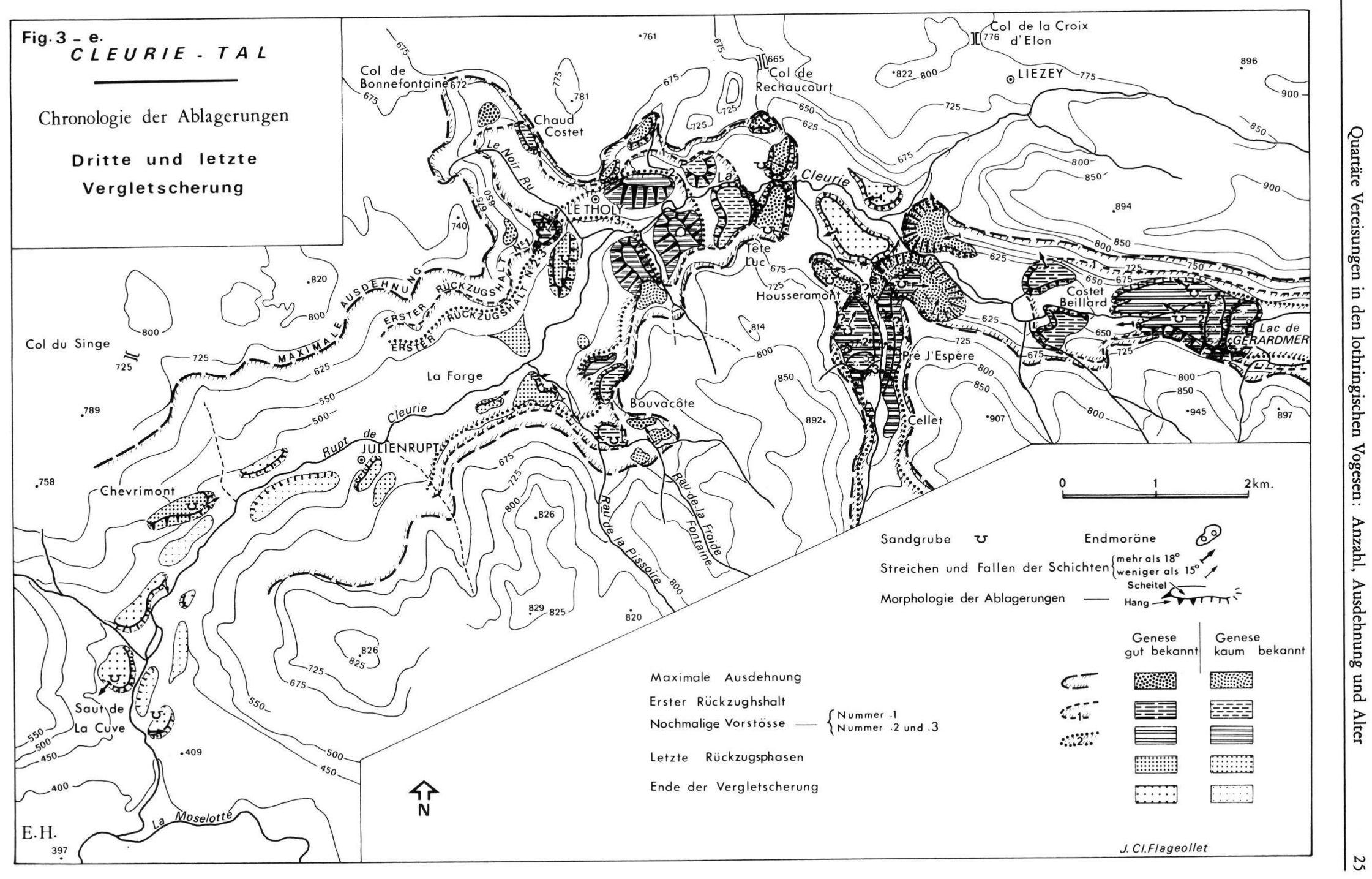


- sedimentologische Argumente: Es gibt zahlreiche lakustrine Delta-Ablagerungen, deren Einfallen den oben genannten Auswölbungsrichtungen entspricht.

- petrographische Argumente: In den Ablagerungen paßt die Verteilung der Gerölle aus Graniten tieferer Bereiche, Graniten der Gratregion, Rhyoliten etc. zu einem dreifachen Ursprung der Eismassen (SERET 1967; FlageOllet \& HAMEURT 1971; FlageOliet 1984).

\subsubsection{Diese drei Eisloben und die zugehörigen \\ Ablagerungen gehören einer einzigen, der dritten Vereisung $(\mathrm{Y})$, an.}

In der Tat ist einerseits der Grad der Verwitterung in allen Ablagerungen schwach und erlaubt es nicht, einzelne einer älteren Vereisung zuzuordnen (SALOME 1968; FlaGeOllet \& HAMEURT 1971). Andererseits war das Tal bei der Vereisung X vollständig von Eismassen erfüllt: Diese überflossen nach $\mathrm{N}$ die Sättel: Croix d'Elon, Rechaucourt, Bonne Fontaine, Singe, und haben Diffluenzen bewirkt, die für die Moränen und die Decken X (s. o.) verantwortlich sind.

\subsubsection{Die Ablagerungen belegen Schwankungen der Gletscherstirn während der maximalen Ausdehnung der dritten Vereisung.}

Für die Details, die die Beschreibungen der Ablagerungen betreffen, siehe FLAGEOLLET \& HAMEURT 1971. Seit dieser Zeit haben eine nochmalige Überprüfung der Morphologie der Ablagerungen von Cellet und die Inbetriebnahme des großen Steinbruches von Housseramont es erlaubt, diesen „End-Komplex” mit einem lakustrinen Delta in genetischer Hinsicht dem von Gérardmer näherzubringen. Ihrer gleichen Höhenlage wegen sind sie als zeitgleich anzusehen.

Jeder Eislobus besitzt die gleiche Zahl von Endmoränenbögen wie Noirgeux. Deshalb wollen wir eine Korrelation zwischen den beiden Gebieten versuchen und wenigstens die wichtigsten Ablagerungen der Cleurie in die maximale Ausdehnung der dritten Vereisung nach dem Schema in Abb. 3 stellen. Dabei übernehmen wir die Interpretation von SERET hinsichtlich der Bildung eines Sees, dessen Niveau durch den Sattel von Rechaucourt reguliert wurde (SERET 1967: 470). Aber es gab nur einen einzigen See mit zunächst geringer, später weiterer Ausdehnung während einer einheitlichen dritten Vereisung.

Glazial-tektonische Spuren kommen in den Moränen und in den lakustrinen Eisrandablagerungen in Cellet, Rain Brice, Beillard häufig vor (FLAGEOLLET \&
HAMEURT 1971); sie belegen kleinere Vorstöße der Gletscherstirn. Deshalb scheinen uns diese Endmoränen an Phasen oder Stadien des Wiedervorstoßens der Gletscherstirn im Laufe eines insgesamt leichten Rückzuges geknüpft, was die Ausdehnung des Sees erklärt.

\subsubsection{Art und Weise des Rückzugs der Diffluenz durch den Moselotte-Gletscher}

Sie können auf verschiedene Art interpretiert werden. Sei es mit Halten während des Rückzugs, erneuten Vorstößen und dem sukzessiven Aufbau von Endmoränen $(\mathrm{Abb} .3 \mathrm{c})$, sei es mit dem Fortbestehen von Toteisresten und der Ablagerung von Kames-Rücken oder -Terrassen (Abb. 3d).

\subsection{Das obere Ognontal zwischen Ternuay und dem Col des Croix}

Im oberen Talabschnitt der Ognon s. s. und in denen ihrer Zuflüsse (Vannoise, Doue de l'Eau) wurden Formen und Ablagerungen von SERET (1967: 216 Fig. 8) und von THEOBALD (Geologische Karte von Frankreich 1:50.000, Blätter Giromagny und Lure) unterschiedlich interpretiert.

SERET hat nach Untersuchung dieses Gebietes weitreichende Schlußfolgerungen gezogen: Der Moselgletscher habe im Würm den Col des Croix überflossen, habe aber nicht bis jenseits des Servance-Riegels gereicht, und die Moränen von Montessaux und Melisey seien rißzeitlich. Die Frage des Zusammenhangs zwischen der zweiten und dritten Vereisung bzw. ihre Übereinstimmung mit Riß und Würm der Alpen wird später aufgegriffen (s. u., Kap. 3). Vorerst ist es wichtig zu wissen, ob und bis an welche Stelle der Moselgletscher der dritten Vereisung in das Ognontal gereicht hat. Zur Stützung der einen oder anderen These wurden zahlreiche Argumente zusammengetragen, die von Beobachtungen bei Mélisey und unterhalb davon ausgehen. Ich möchte hier nur die Argumente überprüfen, die von SERET zugunsten eines würmeiszeitlichen Gletscherhaltes bei Servance vorgebracht wurden. Mit fast zwanzig Jahren Abstand verfügen wir nicht mehr über die gleichen Aufschlüsse wie SERET. Sie sind stark verfallen oder ganz verschwunden. Neue Aufschlüsse gibt es kaum. Dies ist ein weiterer Grund, der Morphologie dieses Gebietes viel Beachtung zu schenken.

\subsubsection{Interpretation der Ablagerungen aus dem Bereich Croix du-Rouille-Evaudois}

Das Tal ist in der Tat durch Ablagerungen abgeschlossen, die sich auf einen Teilriegel stützen und ihm 


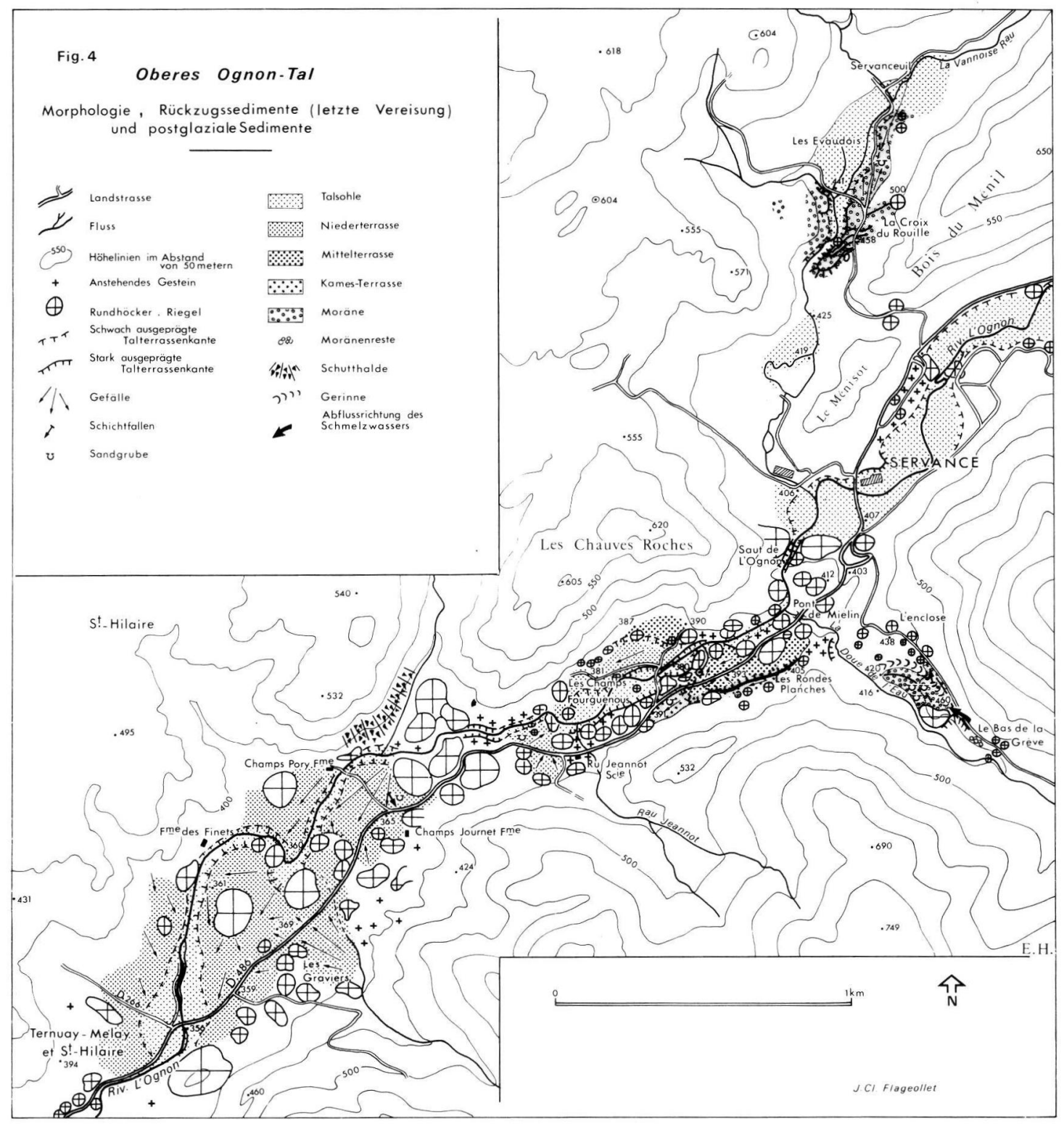

Abb. 4: Oberes Ognon-Tal. Morphologie, Rückzugssedimente (letzte Vereisung) und postglaziale Sedimente.

aufsitzen. Das Abschätzen der genauen Mächtigkeit der Ablagerung ist schwierig und ihre Morphologie ist zweideutig. Es gibt tatsächlich einen Rücken vom Typ Moränenbogen bei Croix du Rouille, von leicht gewundener, konvexer Form, der nach unterhalb des Tales einerseits und nach unterhalb der Straße andererseits getichtet ist. Dieser Bogen ( $458 \mathrm{~m}$ ü. NN bei Croix) setzt sich in zwei Terrassen fort, je eine talaufund talabwärts, letztere etwa zwei bis drei Meter tiefer gelegen. So gut auch das Bild eines Moränenbogens paßt, der talaufwärts gerichtet ist und sich in einem sublakustrinen, eisrandnahen Delta fortsetzt (SERET 1967: 216, Fig. 8), wird es nicht wirklich durch die morphologische Untersuchung bestätigt. In einer stark verstürzten, kleinen Sandgrube zwischen der Kreuzung von Croix du Rouille und der Brücke von Servanceuil ist das Einfallen der kaum geschichteten Sande und Kiese nur schwach und schlecht abzuschätzen. Bei der Brücke von Servanceuil legen die Hangform und das Vorhandensein großer Granitwollsäcke und -blöcke die Existenz von Resten eines Moränenbogens nahe. Dieser stützt sich auf einen Felsriegel 
und ist talabwärts gerichtet. Aufgebaut wurde er durch einen im Vannoisetal herabgeflossenen Gletscher. Die gleiche Interpretation drängt sich für das Gebiet östlich und oberhalb des Gehöftes von Croix du Rouille auf. Als Schlußfolgerung kann der Interpretation von SERET, daß durch Diffluenz des Ognongletschers, der am Gegenhang des Vannoisetales wieder aufgestiegen ist, eine Moräne aufgebaut wurde, nicht wirklich widersprochen werden. Sie wird aber durch die Morphologie der Ablagerungen auch nicht gestützt.

\subsubsection{Das untere Miellintal}

Auf dem rechten Talhang, auf halbem Wege zwischen Bas de la Grève und Enclose, zeigt sich die Akkumulation entlang der D133 wie folgt: Der Bach biegt in einem scharfen Knick nach links, weil er von einem Felsriegel abgelenkt wird, der das Tal teilweise abriegelt. Zwei oder drei senkrechte Felswände erheben sich stufenweise um ca. $25 \mathrm{~m}$ Höhe. Dieser Riegel ist von Sand und Kies überdeckt und wird durch ihn talabwärts verlängert. Sein Scheitel ist auf ca. $150 \mathrm{~m}$ Länge ungefähr eben und endet danach konvexbogenförmig talabwärts gewendet, fällt schräg ab und trifft ca. $200 \mathrm{~m}$ weiter wieder auf den Fluß. Eine Rinne trennt den Riegel vom Hang. Sie beginnt unmittelbar am Anfang der Akkumulation in Hangnähe. Einige Abbruchnischen zeigen einen stark ausgewaschenen Grobsand mit gut gerundeten, manchmal recht groben Geröllen aus Granit und Rhyolit; auf den ersten Metern ist die Ablagerung wenig standfest, sehr frisch und weder gefärbt noch eisenhaltig. Auf der Innenflanke und auf dem Scheitel der Akkumulation sind wechselnde Einfallsrichtungen angedeutet, wobei die Mehrzahl nach WNW gerichtet ist, d.h. talabwärts und zur Talachse hin. Moränenreste (gerundete und kantige Blöcke) treten talaufwärts zwischen diesem Riegel und dem des Bas de la Grève häufiger auf. Diese Befunde führen zu folgender Interpretation der Ablagerung: Es kann sich um die zwischen dem Riegel eingeschlossene Endmoräne eines Gletschers oder um eine durch den Riegel vor nachfolgender Erosion geschützte Kames-Ablagerung handeln. In jedem Fall aber ist die Ablagerung in Verbindung mit einem Gletscher zu sehen, der das Miellintal herabgeflossen war. Die Interpretation von SERET, es handele sich um eine talaufwärts durch ein lakustrines Delta verlängerte Moräne, die beide durch Diffluenz des im Miellintal aufgestiegenen Ognongletschers entstanden sind, scheint mir kaum vereinbar mit der Morphologie der Ablagerung und der des gesamten Gebietes.

\subsubsection{Das Ognontal unterhalb von Servance}

Die Sand- und Schotterablagerungen, die die Rundhöcker überdecken oder ihnen anliegen, sind in den beiden Stammbecken zwischen Servance und Ternuay-Melay und St. Hilaire, dem der Champs Fourguenous und denen der Champs Pory, Journet und les Graviers weit verbreitet. SERET sieht in ihnen eisrandnahe Schüttungen der Diffluenz des Moselgletschers bei seinem würmzeitlichen Halt bei Servance.

\subsubsection{Das Stammbecken der Champs Fourguenous}

Es liegt in einem Gebiet, das von stark durch Brüche gegliederten Felsen geprägt ist, aus dem aber noch zahlreiche Rundhöcker herausragen. Diese sind von sandig-kiesigen Sedimenten bedeckt oder umgeben, die die Topographie ausgleichen, indem sie ihr den ebenen Charakter einer Aufschüttungsfläche geben. Man unterscheidet hier mehrere Höhenniveaus:

- Das schmale Niveau der Rondes Planches auf dem Südhang, mit schwacher Neigung talabwärts.

- Ein Niveau einige Meter unterhalb, zwischen 393 und 387 m Höhe ü. NN gelegen, auf beiden Talflanken.

- Die eigentlichen Champs Fourguenous als Niederterrasse.

- Die Talsohle der Ognon.

Erinnern wir uns, daß SERET die Ablagerungen der Rondes Planches als rißzeitliche Kames-Terrasse und die der Champs Fourguenous als Sanderterrasse des Würm interpretiert hat.

\subsection{Die Rondes Planches}

Ihre Topographie ist die einer unregelmäßigen, talabwärts geneigten, schmalen Terrasse (Breite unter $30 \mathrm{~m}$ ), die durch einen unzusammenhängenden, aber recht gestreckten Hang begrenzt wird. Die Verebnung setzt sich bis zum Punkt 391 ü. NN fort und die Schotter und Sande finden sich im Feld links der Straße. Was die eigentlichen Rondes Planches betrifft, läßt sich nicht mit letzter Sicherheit sagen, daß es sich ausschließlich oder vorwiegend um eine Akkumulationsform handelt; der Rand ist möglicherweise im verwitterten Fels ausgebildet. Falls es sich um eine Akkumulationsform handelt, paßt ihr steil ausgebildeter Hang zu einer eisrandnahen Herkunft, aber am Gegenhang fehlt eine solche Form vollständig. Es wäre einfacher, sie als Kames-Terrasse zu interpretieren als in ihr einen stark erodierten Sander zu sehen. Wie SERET (1967: 21) sagt: "aucune coupe 
n'est susceptible de révêler leur âge" (Kein Aufschluß erlaubt es, ihr Alter zu bestimmen). Aber sie kommen nicht "à l'aval du point de départ des épandages proglaciaires du Würm" (unterhalb des Ausgangspunktes der würmzeitlichen Sanderschüttung) vor (SERET 1967: 217). Der Ursprungsort liegt sehr dicht beim Riegel der Brücke von Miellin. Zusammenfassend denke ich, daß man die genetische Interpretation (als Kames-Terrasse) akzeptieren kann, aber es gibt keine Hinweise auf ein höheres Alter (Riß).

\subsection{Die Champs Fourguenous}

In der Tat sind hier zwei Niederterrassen zu sehen. Sie liegen einige Meter über der heutigen Talsohle der Ognon. Die Hänge zeigen hier ziemlich grobe Schotter, die in stark ausgewaschenen, frischen Sanden gleichmäßig verteilt sind. Handelt es sich um die Reste zweier aufeinanderfolgender Sanderschüttungen, die sich ineinander eingeschachtelt haben? Warum nicht um Kames-Ablagerungen während der Rückzugsphasen der letzten Vereisung, als die Doue de l'Eau und die Ognon weniger stark in den Riegel eingeschnitten waren als sie es heute sind? Dieses Stammbecken setzt sich aus einer Serie kleiner, durch Rundhöcker abgeschlossener Becken zusammen, unter Ausbildung eines gestuften Tallängsprofils. Sie können fast gleichzeitig verfüllt worden sein, als die Schuttlast des Flusses bei gleichzeitig geringerer Eintiefung höher war als heute.

\subsection{Die Kiesgrube am Jeannot-Bach}

Stark verstürzt existiert sie heute noch, und es lassen sich hier zwei Beobachtungen machen:

- Die an der Oberfläche sichtbaren, gut gerundeten Granitgerölle sind hier in sandig-kiesigen Ablagerungen aufgeschlossen.

- Die Sedimente liegen nicht genau an der Mündung des Jeannot-Baches und es ist nicht sicher, ob sie von dorther stammen. Ich sehe sie eher als Fortsetzung der Niederterrasse denn als periglazialen Kegel an.

\subsubsection{Das Stammbecken der Champs Journet-Ternuay}

Zwischen dem Gehöft von Champs Pory und dem von Champs Journet ist die Verfüllung unregelmäßig, aber relativ mächtig ( $\geq=3 \mathrm{~m}$ ). Das schwache Einfallen und die Hangneigung lassen eine Schüttung durch die Ognon vermuten. Der Fluß ist nur schwach unter Ausbildung einer sehr niedrigen Terrasse in diese Decke eingeschnitten, die die der Champs Four- guenous verlängert. Die Kiesakkumulation hat eine nur geringe Ausdehnung und wird von einem großen Rundhöcker begrenzt. Im Gegensatz zu dem, was SERET angibt, glaube ich nicht, daß die Kiesakkumulation bei ihrer Ausbreitung die Ognon nach $\mathrm{N}$ zurückdrängen konnte (SERET 1967: 241). Die Hangfußbereiche nahe des Hofes von Champs Pory wie auch ein Riegel entlang der Straße sind gestreckte Hänge. Sie werden durch eine unzusammenhängende Hangschuttdecke wechselnder Mächtigkeit ausgeglichen, die ihr Material aus leicht verwittertem, rötlichem und stark tektonisch zerrüttetem Gestein (v. a. Rhyolite) bezieht. Man darf daraus nicht ohne genauere Untersuchung schließen, daß das Vorhandensein von rötlichem Ton ein wie auch immer höheres Alter des Schuttes anzeigt, und daß es sich um periglaziale Schuttdecken handelt. Der Zustand des Materials ist dergestalt, daß der Schutt - heute im allgemeinen festliegend - nicht unbedingt in einem sehr kalten Milieu abgelagert worden sein muß. Mit anderen Worten, er kann sehr jung sein, während des Gletscherrückzuges abgelagert und nicht unbedingt "contemporains du maximum du froid" (zeitgleich mit dem Kältemaximum) (SERET 1967: 241). Die oberen Talabschnitte von Vologne, Mosel und Moselotte, die erst später eisfrei wurden, sind an manchen Stellen mit dem gleichen Schutt überdeckt, für dessen Genese der tektonische und petrographische Faktor eine ebenso große, wenn nicht größere Rolle spielte als das Klima.

\subsubsection{Schluß}

Im oberen Ognontal verfüllten mächtige Sedimente den Boden der glazialen Stammbecken oberhalb von Servance. Sie können eisrandnahen Ursprungs sein, am äußersten Ende eines Talgletschers abgelagert, der durch das Überfließen des Moselgletschers zustande kam. Dieser Gletscher konnte eine kurze Diffluenz verursachen und das Vannoisetal bei Evaudois plombieren. Ich kann diese Interpretation von SERET weder bestätigen noch widerlegen. Bestimmt gab es noch einen Gletscher im Tal der Doue de l'Eau, dessen äußerstes Ende ein wenig oberhalb von Enclose oder beim Riegel von Servance lag.

Die Argumente zur Anbindung dieser Situation an das Würmhochglazial von (SERET 1967) sind anfechtbar. Ebenso gut konnten diese Akkumulationen während der Rückzugsphasen der letzten Vereisung entstanden sein und m. E. kann sich der Eishalt bei Servance auch während des Abschmelzens eingestellt haben.

Die sehr charakteristische morphologische Gliederung des Tales in Becken und Felsschwellen muß zu einem ruckhaften Gletscherrückzug geführt haben, mit 


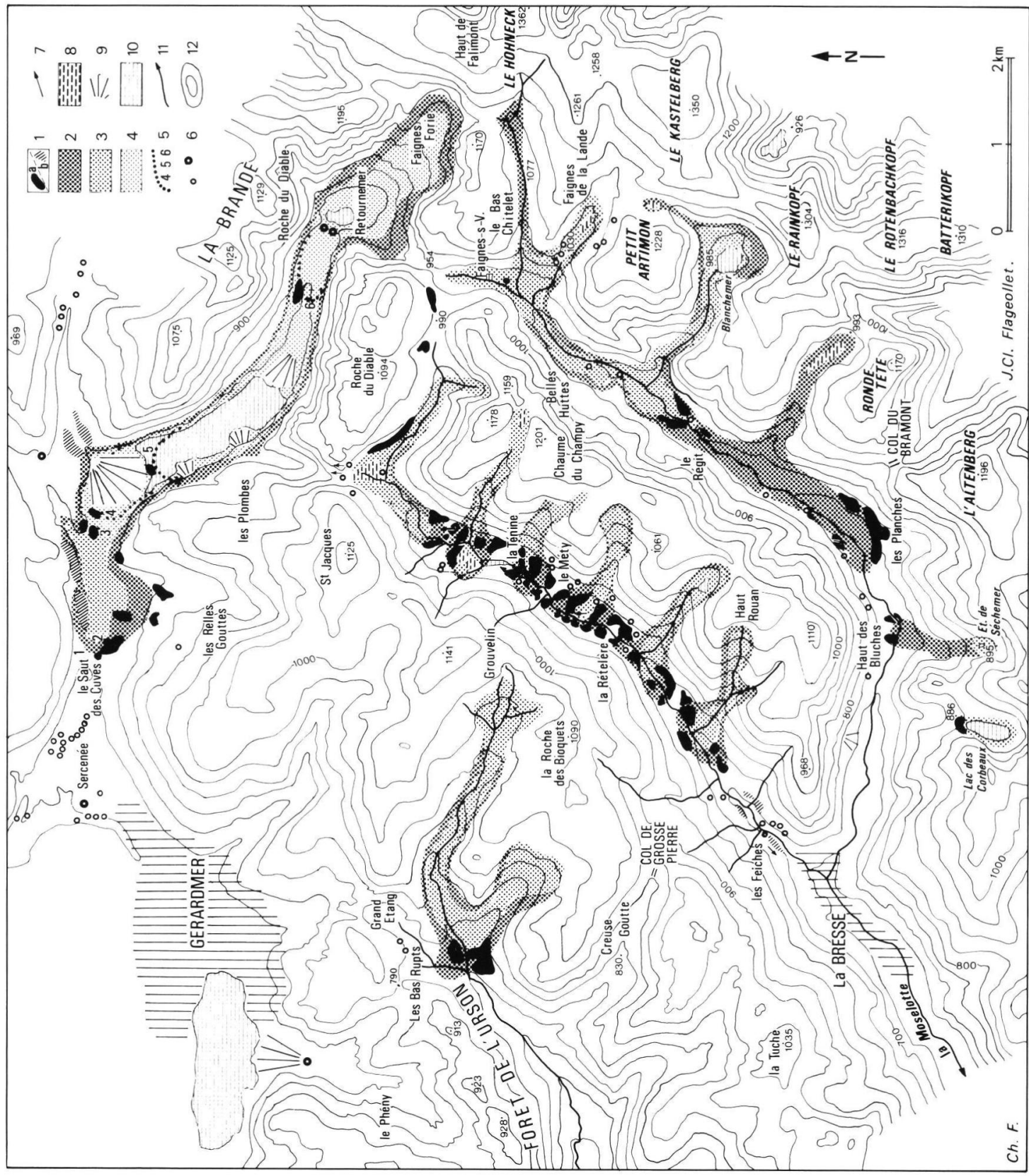

Abb. 5: Letzte Vereisung. Letzte nochmalige Vorstöße in den oberen Talabschnitten von Vologne und Moselotte.

\section{1 a Endmoränen}

$1 \mathrm{~b}$ Fluvioglaziale Terrasse

2 Ausdehnung der Gletscher. Erste Phase

3 Ausdehnung der Gletscher. Zweite Phase

4 Letzte nochmalige Vorstöße

5 Letzte nochmalige Vorstöße 3,4, 5 und 6

6 Riegel, Rundhöcker
7 Schichtfallen

8 Torfmoor

9 Lakustrines Delta, Schwemmfächer

10 See

11 Fluß

12 Höhenlinien 
einem stark verspäteten Abschmelzen oberhalb des Riegels von Servance und Toteisblöcken, die einige Zeit im tiefsten Becken direkt unterhalb erhalten blieben.

\subsection{Obere Moselotte und obere Vologne}

Oberhalb von Saut des Cuves, im E von Gerardmer und an den beiden Armen der Moselotte, den parallelen Tälern der Moselotte und des Chajoux-Baches, treten glaziale Ablagerungen im Volognetal häufig auf. Die Fragen, die sie aufgeworfen haben, sind vor allem genetischer Natur: Endmoränen, Kames-Rücken oder Kames-Terrassen und nicht so sehr stratigraphischer Art. Alle Autoren betrachten sie als sehr jung, zeitgleich mit dem Ende der letzten Vereisung. Diese beiden Fragen sind aber eng miteinander verknüpft. In der Tat kann man ihnen, wie mehrere Autoren es tun, einen gewissen stratigraphischen Wert zusprechen, bei dem jede Endmoräne einem Halt oder einem erneuten Vorstoß des Gletschers während des Abschmelzens entspricht. Und in diesem Fall muß man zu erfahren versuchen, ob sich diese Phasen mit pollenanalytischen Befunden in Bezug auf Klimaschwankungen korrelieren lassen (s. u., Kap. 3). Oder man darf ihnen eben keinerlei stratigraphischen Wert zuerkennen, wenn ihre Genese als gemeinsam und gleichzeitig angesehen wird, wie SALOME (1968: 57) es vorgeschlagen hat.

Die unterschiedlichen Interpretationsrichtungen, die die Genese der Ablagerungen betreffen, waren vor allem im Chajouxtal spürbar. Seit 1971 habe ich in Abständen immer wieder die Ablagerungen dieses Tals im Wandel der Aufschlußsituation und entsprechend des Fortschreitens der wissenschaftlichen Arbeiten untersucht. Die Ergebnisse sind in Abb. 5 zusammenfassend dargestellt.

Die Mehrzahl der Ablagerungen von Chajoux und Mosel sind Moränen und keine Kames-Rücken. Die überwiegende Strukturlosigkeit, die Heterometrie und steil einfallende Schichten in kleinen, lakustrinen Sedimentbecken beweisen es; sie sitzen nicht oder nur selten Felsriegeln auf. Es handelt sich aber nicht um durchgehende Moränenbögen. Diese Moränen sind häufig stark aufgelöst, und diese Auflösung ist teils syngenetisch, teils postgenetisch als Folge der Eintiefung des Flusses bedingt. Im Chajouxtal sind diese Moränen $\mathrm{zu}$ vielgestaltig, $\mathrm{zu}$ zahlreich und $\mathrm{zu}$ dicht beieinander liegend, als daß man jede von ihnen mit einer Gletscherschwankung in Verbindung bringen könnte. Dies gilt vor allem für dieses Tal, wo es oberhalb kein hochgelegenes Kar gibt (Lispachsee). Außerdem sind die Einfallswinkel mancher Ablagerungen talaufwärts gerichtet (GUEBOURG 1972; RAHM
1977). Das unterstützt die Vorstellung von EGGERS (1964: 58), nach der diese Moränen von durch das Tal abfließenden Gletschern abgesetzt wurden, die parallel und gleichzeitig von benachbarten Karen ausgingen.

\section{Glazialstratigraphie der Vogesen}

\subsection{Welche Stratigraphie gilt für die Vogesen?}

Bis in die letzten Jahre wurde in den Vogesen die letzte nachgewiesene Vereisung dem Würm der alpinen Stratigraphie gleichgesetzt. Dies geschah aufgrund der Frische der Ablagerungen und wegen des späten Abtauens der letzten Kare in den Talschlüssen (FlaGeoliet 1980). SALOME (1967) und SerET (1968) haben sich weiterhin auf diese Stratigraphie bezogen. Es ist noch nicht lange her, daß im benachbarten Jura CAMPY (1982) zwei aufeinanderfolgende Vereisungen nachgewiesen und diese mit Riß und Würm in Beziehung gebracht hat. Die Arbeiten von WOILLARD (1973 bis 1978) haben sich gleichermaßen an der westeuropäischen Nomenklatur von WOLSTEDT und MENKE orientiert, die von der Gleichzeitigkeit der alpinen und der norddeutschen Vereisungen ausgeht. Seine Arbeiten haben aber mehrere Neuerungen eingeführt:

- Korrelation mit Schwankungen der Paläotemperatur, die an Tiefseesedimenten festgestellt wurden, sowie absolute Datierungen.

- Erstellung einer Biostratigraphie, die eine lokale Nomenklatur (Mélisey, Saint-Germain, Lanterne etc.) benützt und schließlich die Aufgabe jeden Bezuges zur alpinen stratigraphischen Terminologie (WOILLARD 1978: 16).

Die Frage der Gleichzeitigkeit zwischen den alpinen und den nordischen Vereisungen war außerhalb der Vogesen heftig umstritten und die Zahl dieser Vereisungen wurde ebenfalls erneut in Frage gestellt. Viele Forscher haben es vorgezogen, sich wieder auf lokale Litho- und Chronostratigraphien zu berufen, und in den Vogesen hat SERET aufgehört, die Begriffe Mindel, Riß und Würm zu benutzen. Dies ist ein Zeichen vorsichtigen, auf jeden Fall aber nur vorläufigen Vorgehens, weil noch kein Forscher darauf verzichtet hat, Korrelationen mit den europäischen Bio- und Lithostratigraphien herzustellen.

\subsection{Die Bedeutung der Endmoränen in der Stratigraphie}

Man verfügt einerseits über die relative, bio-klimatische Stratigraphie und über absolute Datierungen, 
ausgearbeitet für Grand Pile, andererseits hat man gestufte Moränen in den Tälern. Kann man eine Annäherung zwischen den Eisrandlagen anhand der Moränen und den durch die Pollenanalysen aufgezeigten Klimaschwankungen versuchen?

Die Antwort fällt nicht leicht, und viele Autoren haben sich gefragt, wie es VEYRET (1981: 297) in Erinnerung ruft, in welchem Maße nicht die mehrfach versuchten Korrelationen zwischen den Moränenbögen und möglichen Klimaschwankungen allzu oft anfechtbaren Schlußfolgerungen Raum gegeben haben. An Einwänden gegen diese Art von Korrelation fehlt es nicht: Im Gebirge sind einige rezente Gletscher im Anwachsen begriffen, während andere sich zurückziehen. In einigen Jahren können sich vor den alpinen Gletschern zahlreiche Moränenbögen aufbauen, die nichts weiter als kleinere Phasen innerhalb klimatischer Änderungen aufzeigen. Manche Stadien, Rückzugshalte oder nochmalige Vorstöße sind nicht notwendigerweise durch Interstadiale getrennt, während denen eine Klimaänderung einen deutlichen Rückgang der Gletscher bewirkt hätte. Wie soll man unterscheiden zwischen dem, was auf eine wirkliche Klimaverschlechterung zurückgeht und dem, was aus einem kleinen Gletscherzuwachs aufgrund geringer Klimamodifikation resultiert?

\subsection{Moränen der Maximalausdehnung der dritten Vereisung und ihre Chronologie}

Während der maximalen Ausdehnung waren die oberen Talabschnitte alle von Eismassen erfüllt. Dabei bestanden viele Zusammenflüsse und zahlreiche Verbindungen waren von Eis bedeckt. Unter Berücksichtigung dieser Tatsache kann man dem zustimmen, daß die gesamte Eismasse bis in ihre Randbereiche hinein in weltweitem Maßstab reagierte, und daß sie andererseits nur auf Klimaänderungen gewissen Umfangs reagierte. Unter dieser Prämisse kann man nun verstehen, was bei Noirgeux, Tholy und Remiremont festgestellt wurde und was gleichermaßen auch bei Mélisey-Montessaux vorkommt: Dicht beieinanderliegende Endmoränenbögen, die fast überall in Vierzahl entwickelt sind (Abb. 6a). Ferner kann man eine Parallelisierung mit der Klimaentwicklung von Grand Pile versuchen. Wo kann der Gletschervorstoß im Pollendiagramm von Grand Pile angesiedelt sein, der für die Moränen von Montessaux und Mélisey verantwortlich ist?

- Woillard (1978: 16) blieb dem Schema von SERET treu. Für sie sind Mélisey I und Mélisey II des Pollendiagramms zeitgleich mit der mittleren Vereisung (Riß I und Riß II) und somit älter als die letzte Vogesenvereisung (Lanterne), während der der Gletscher im Ognontal nicht mehr über Servance hinaus- reichte. Neben den bereits oben genannten Beobachtungen über die Interpretation der Ablagerungen aus dem Raum Servance lassen andere Gründe an dieser Korrelation zweifeln und eine andere Lösung vorziehen.

Die Kaltzeiten Mélisey I und Mélisey II sind kürzer und deutlich weniger kalt als Lanterne (Abb. 6b). Das führt folglich nach dem Schema von SERET zu der Aussage, daß der Moselgletscher, der in das Ognontal übertrat, während der kurzen und gemäßigten Kaltphasen mächtig war und weit gereicht hat (bis Montessaux-Mélisey), und daß er während der langen und intensiveren letzten Kaltzeit (Lanterne) weniger mächtig und weniger weitreichend war (Servance). Dies ist durch die Tatsache, daß bedeutende Gletscher während Mélisey I und II nicht sicher nachgewiesen sind, ein schwerwiegender Widerspruch. Nach BEAU. LIEU \& REILLE (1984: 8) handelt es sich um kurze, voneinander getrennte Kälteperioden während längerer Erwärmungsphasen (St. Germain), die, wie bei Les Echets, nur gemäßigte Phasen sind, welche nicht den bio-klimatischen Status von Interglazialen besitzen.

- Es scheint einleuchtender, eine Annäherung der Moränen von Mélisey an die Glazialstadien von Lanterne II und III vorzunehmen (Abb. 6b).

Lanterne III ist indessen kälter und längerdauernd als die vorhergehenden Stadien. Wie also soll man erklären, daß die Gletscher überall von Stadium I zu Stadium IV leicht zurückgewichen sind? Die Erklärung liegt vielleicht in der Tatsache, daß es bei stärkerer Abkühlung zum Ende des Lanterne auch trockener war, was eine leichte Abnahme des Gletschervolumens mit sich bringen konnte.

\subsection{Rückzugsmoränen der oberen Vologne} und der oberen Moselotte und ihre Stratigraphie

Im Unterschied zu Moränen der maximalen Vereisung sind die des Abschmelzens eher von lokalen Klimabedingungen und von kurzfristigen und unbedeutenderen Klimaänderungen abhängig. So kann man verstehen, daß die Zahl der Stadien in der oberen Moselotte und der oberen Vologne unterschiedlich ist. Ohne ein einziges tiefergelegenes, ausgedehntes $\mathrm{Kar}$ gibt es ein NW-orientiertes Tal etc. und dies in Verbindung mit einem unterschiedlichen Glazialregime. Dagegen muß in den beiden Paralleltälern der oberen Moselotte und des Chajoux das Regime aus mehreren Gründen das gleiche geblieben sein: Gleiche Exposition, gleiche Orientierung der Täler, gleiche Morphologie und Längserstreckung der Eiszungen, d. h. gleicher Umfang des erneuten Vorrückens, leicht variierend nach Höhenlage und Größe der Kare. Von dorther kommen die wichtigsten Eiszungen, die 


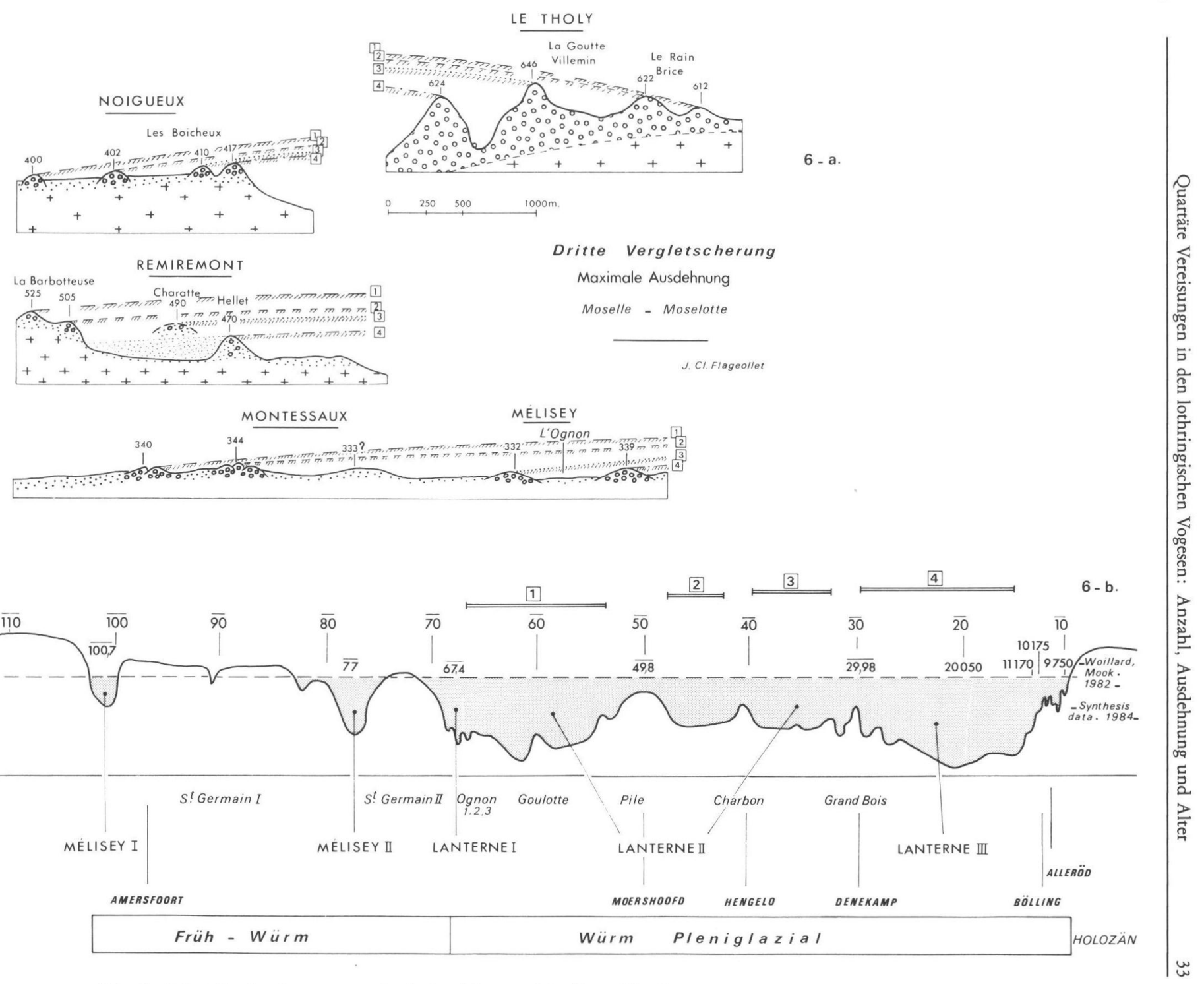

Abb. 6: Dritte Vergletscherung, maximale Ausdehnung. Moselle - Moselotte. 


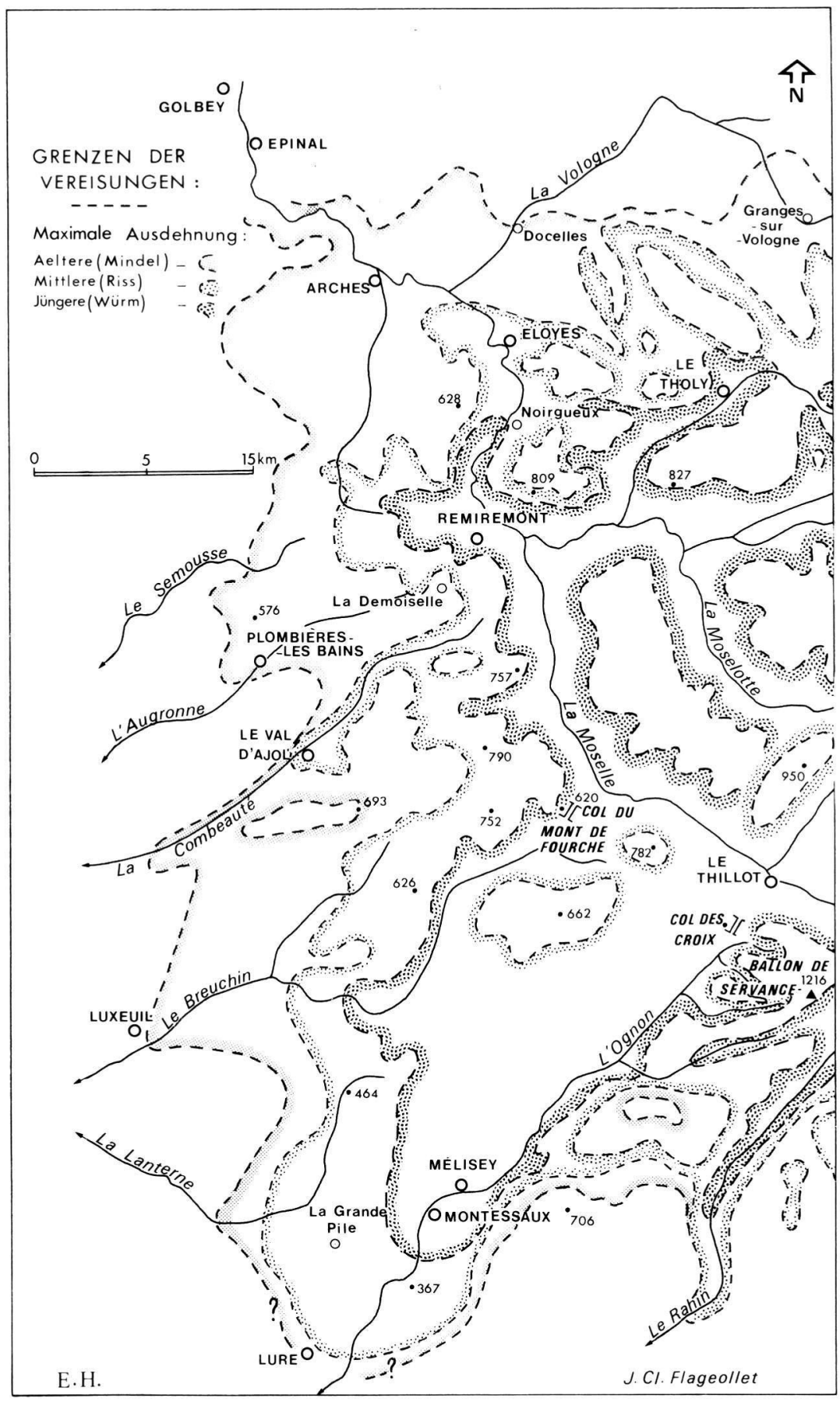

Abb. 7: Ausdehnung der eiszeitlichen Vergletscherungen in den lothringischen Vogesen. Moselbecken. 
Zusammenflüsse und die weniger zahlreichen, aber umfangreicheren Ablagerungen im Moselottetal, das am dichtesten an den Vogesenhauptkamm heranreicht.

In diesem Raum scheint es zwei nochmalige Vorstöße gegeben zu haben.

Welchen Abkühlungsphasen soll man sie zuordnen? Vielleicht der älteren und mittleren Dryaszeit. In der Tat wurde der Teich von Machey sehr spät eisfrei (JANSEN 1974) und die Karte der oberen Vologne sind in Höhenlage und Größe dem Kar von Frère Joseph vergleichbar, wo das Abtauen im Alleröd begann (SERET 1984).

\section{Schluß}

Die Vereisung der mittleren lothringischen Vogesen vollzog sich in mindestens drei Schüben und jede der aufeinanderfolgenden Vereisungen war weniger umfangreich als die vorausgehende. Im Lauf der letzten endete der Moselgletscher bei Noirgeux, trat nach Süden aus dem Tal in den Raum Le Thillot aus, und schob sich im Ognontal bis in den Raum Mélisey vor (Abb. 7).

Nach den Korrelationen, die man nach der bio-klimatischen Stratigraphie des Grand Pile und den Moränen, die die maximale Ausdehnung kennzeichnen, aufstellen kann, blieb sie zwischen den Kaltphasen des Lanterne, d. h. während des ganzen Würmhochglazials bis zum Ende des Lanterne III bestehen.

Aufgrund der gleichartigen Stratigraphie von Grand Pile und den Befunden von Les Echets ist man doch geneigt, sich nach der alpinen Stratigraphie zu richten. Die letzte Vereisung der mittleren lothringischen Vogesen (Lanterne in der Chronologie des Grand Pile) fand also während des Würm statt. Der Aufbau der Gletscher muß ab 70.000 B.P. sehr schnell vor sich gegangen sein und erreichte seinen Höchststand um 67.000 B.P. Der rasche Rückzug begann ca. 15.000 B.P. Die beiden vorhergehenden Vereisungen können demnach weiterhin in Riß und Mindel gestellt werden.

Herzlichen Dank an B. SpONHOLZ und R. KuRz für die deutsche Übersetzung dieser Arbeit.

\section{Schriftenverzeichnis}

DE Beaulieu, J. L. \& Reille, M. (1984): The pollen sequence of Les Echets (France). A new element for the chronology of the upper pleistocene. - Geogr. phys. et quaternaire, XXXVIII, 1: 3-9, 2 Abb.; Montréal.
Caillier, M. (1977): Etude chronoséquentielle des sols sur terrasses alluviales de la Moselle, Genèse et évolution des sols lessivés glossiques. - UER STMCM, Université de Nancy, I: 87 S., 27 Abb., 10 Fot.; Nancy.

CAMPY, M. (1982): Le Quaternaire franc-comtois. Thèse Doctorat Sciences Naturelles. - 575 S., 222 Abb.; Besançon (Faculté des Sciences).

CAPOT-Rey, R. (1938): La structure et le relief. In: Geographie lorraine, II: 41.107, Nancy (Berger-Levrault).

EgGERS, H. (1964): Schwarzwald und Vogesen. Ein vergleichender Überblick. - Westermann Taschenbuch. Geographie. 1: 144 S., 6 Kt., 5 Abb.; Braunschweig (Westermann).

Flageollet, J. C. (1976): Itinéraire 19. Les formations quaternaires entre la Schlucht et Remiremont. - In: Guide Géologique Vosges: Alsace: 123-129; Paris (Masson).

- (1979): Itinéraire 9: Morphologie et accumulations quaternaires de la vallée de la Moselle à la sortie des Vosges. - In: Guide Géologique Lorraine-Champagne: Paris (Masson).

- (1980): Chronostratigraphie dans les terrasses alluviales de la Moselle à la sortie du massif vosgien. - In: Problèmes de stratigraphie quaternaire en France et dans les pays limitrophes. Supplt Bull. A.F.E.Q. 1: 110-113, Paris.

- (1984a): La terrasse würmienne à l'aval de la moraine de Noirgeux entre Remiremont et Epinal (Vosges): signification dynamique et conditions paléoclimatiques. Bull. Assoc. Franç. Etude du Quaternaire, 1/2/3: 93-98; Paris.

- (1984b): Cent cinquante ans de recherches sur le glaciaire vosgien. Historique des idées et état de la question. - in Mélanges offerts au Professeur ANDRÉ JOURNAUX: 173-192, 1 Kt.; Caen (Université de Caen).

- \& HameurT, J. (1971): Les accumulations glaciaires de la vallée de la Cleurie (Vosges). - Revue Géogr. de l'Est: 119-181, 7 Abb., 4 phot.; Nancy.

Guebourg, J. L. (1972): Etude géomorphologique de la vallée du Chajoux (La Bresse, Vosges). - Mém. maîtrise géogr. phys., Univ. de Nancy II; Nancy.

JANSEN, C. R. (1974): Oversicht van het paleobotanisch onderzoek in de Vogesen en enkele op merkingen over de synchroniteit van pollen zones. - Vogezen symposion 1974, Institut voor Bodemkunde, 37-47, 5 Abb.; Utrecht.

LOMBARD, G. (1977): Morphologie et accumulations glaciaires sur le plateau du Fossard (Vosges, France). Mém. maitrise géogr. phys., Univ. de Nancy II, Nancy.

MaIre, A. (1972): Etude géomorphologique de la Haute Moselotte en amont de la Bresse (Vosges, France). Mém. maîtrise géogr. phys., Univ. de Nancy II; Nancy.

MeYer, L. (1911-1913): Les Vosges méridionales à l'époque glaciaire. - Mitteilungen der Naturhistorischen Gesellschaft in Colmar, XI, XII: 1-135; Colmar. 
Nordon, A. (1931): Etude des formes glaciaires et des dépôts glaciaires et fluvioglaciaires du bassin de la HauteMoselle. - Bull. Soc. Géol. de France, V, 1: 245-288, 23 Abb.; Paris.

RAHM, G. (1977): Eine Stauchendmoräne und andere Stauchungserscheinungen in Glazialtälern der Südvogesen. - Ber. Naturf. Ges., 67: 249-253, 3 Abb.; Freiburg im $\mathrm{Br}$.

Salome, A. I. (1968): A geomorphological study of the drainage area of the Moselotte and upper Vologne in the Vosges (France). - 98 S., 17 Abb., 4 Kt.; Utrecht.

Seret, G. (1967): Les systèmes glaciaires du Bassin de la Moselle et leurs enseignements. - Revue Belge de Géographie, 2, 3: 577 S., 76 Abb., Bruxelles.

- (1984): Some aspects of glaciations in the Vosges lorraines. - In: Dynamical and chronological relations between glacial and periglacial deposits. Int. Union for Quatern. Research. S. 25-45. Centre de Géodynamique sédimentaire et Evolution géobiologique. LA. CNRS 157, Dijon.

Service Geologique National (1974): Carte géologique détaillée de la France au 1/50000, feuille GIROMAGNY, Orléans.

- (1976): Carte géologique détaillée de la France au $1 / 50000$, feuille MuNSTER, Orléans.

- (1978): Carte géologique détaillée de la France au 1/50000, feuille GÉrardmer, Orléans.

- (1979): Carte géologique de la France au 1/50000, feuille REMiremont, Orléans.

THeobald, N. (1969): Chronologie des dépôts quaternaires le long de la bordure méridionale des Vosges. - Annales Scientif., Univ. de Besançon, 3e série, géol., 6: 23-28, 1 Abb.; Besançon.
Theobald, N. (1973): Dépôts glaciaires, fluvioglaciaires et fluviatiles de la retombée méridionale des Vosges. Annales Scientif., Univ. de Besançon, 3e série, géol. $\mathrm{n}^{\circ}$ 19 bis, 257-266, 4 Abb.; Besançon.

VAskou, Ph. (1981): Apport de la sédimentologie à la connaissance des dépôts alluviaux des terrasses de la Moselle, de Noirgueux à Toul. - $145 \mathrm{~S}$. Thèse $3 \mathrm{e}$ cycle, Univ. de Nancy I, Nancy.

- (1984): La Terrasse moyenne de la Moselle dans les Vosges; essai de reconstitution de la genèse d'une terrasse fluviatile par rapport au cycle climatique quaternaire. - Bull. Association Franç. Etude du Quatern., 1/2/3: 99-103, 3 Abb.; Paris.

VEYRET, Y. (1981): Les modelés et formations d'origine glaciaire dans le Massif Central français. - Thèse Paris, 2 Bd.: 338 S., 68 Abb.; Lille (Presses Universitaires).

Wolllard, G. (1975): Recherches palynologiques sur le Pléistocène dans l'Est de la Belgique et dans les Vosges lorraines. - Acta geographica Lovaniensia, 14: $168 \mathrm{~S}$.; Louvain.

- (1978): Grande Pile Peat Bog: A continuous Pollen Record for the last 140,00 years. - Quaternary Research, 9: 1-21, 5 Abb.; Washington.

- \& MooK, W. (1982): Carbon-14 Dates at Grande Pile: Correlations of land and sea chronologies. - Science 215: 159-161; Washington.

Gérardmer, Weihnachten 1985. 